\title{
The Different Association between Metformin and Sulfony- lurea Derivatives and the Risk of Cancer May be Confounded by Body Mass Index
}

\author{
Catherine E. de Keyser ${ }^{1,2}$, Loes E. Visser ${ }^{1}$, Albert Hofman ${ }^{1}$, Bruno H. Stricker ${ }^{1,2^{*}}$, Rikje Ruiter ${ }^{1 \#}$ \\ ${ }^{1}$ Department of Epidemiology, Erasmus Medical Center, Rotterdam, the Netherlands \\ ${ }^{2}$ The Health Care Inspectorate, The Hague, the Netherlands
}

"Corresponding author: Bruno H. Stricker, Department of Epidemiology, Erasmus MC, P.O. Box 2040, 3000CA Rotterdam, the Netherlands, Tel: +31-10-7044958; Fax: +31-10-7044657; E-mail: b.stricker@erasmusmc.nl

\#Submitting author: Rikje Ruiter, Department of Epidemiology, Erasmus MC, P.O. Box 2040, 3000CA Rotterdam, the Netherlands, Tel: +31-10-7044958; Fax: +31-10-7044657; E-mail: c.dekeyser@erasmusmc.nl

\begin{abstract}
Aim: Several studies in large databases suggest that in comparison to glucose-lowering sulfonylurea derivatives, metformin is associated with a reduced risk of cancer in patients with diabetes. As many databases miss relevant confounder data, our objective was to investigate whether the determinants age, body mass index (BMI), alcohol consumption, and renal function were associated with dispensing of either metformin or sulfonylurea derivatives as first drug therapy for type 2 diabetes mellitus while taking into account calendar time.

Methods: We identified 639 incident metformin users and 934 incident sulfonylurea derivatives users in the Rotterdam Study, a prospective population-based cohort study. Associations were studied using logistic regression analyses.

Results: After adjustment for all other determinants, starters with metformin had a statistically significantly higher BMI than starters with sulfonylurea derivatives (OR 1.19, 95\% CI $1.04-1.37$ for starters < July $1^{\text {st }} 2000$; OR $1.23,95 \%$ CI 1.04 -1.45 for starters $\geq$ July $1^{\text {st }} 2000$ ). Age, renal function, and alcohol consumption were not statistically significantly associated with the probability of dispensing metformin versus sulfonylurea derivative therapy as first drug therapy for type 2 diabetes mellitus.

Conclusion: BMI is associated with a higher probability of dispensing of metformin as first drug therapy for type 2 diabetes mellitus in comparison to sulfonylurea derivatives. BMI is associated with the risk of cancer as well as the risk of type 2 diabetes mellitus. Therefore, in studies analyzing the association between the use of metformin or sulfonylurea derivatives and the risk of cancer, BMI should be considered as an essential co-variable.
\end{abstract}

Received date: July 09, 2016

Accepted date: : September 05, 2016

Publication date: September 12, 2016

Citation: Stricker, B.H., et al. The Different Association between Metformin and Sulfonylurea Derivatives and the Risk of Cancer May be Confounded by Body Mass Index. (2016) J Diabetes Obes 3(2): 37- 42.

DOI: $10.15436 / 2376-0494.16 .1007$

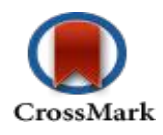

Keywords: Metformin;Sulfonylurea derivatives; Type 2 diabetes mellitus; Cancer; Body mass index 


\section{Introduction}

Type 2 diabetes mellitus is a common disorder of which the prevalence rises, due to factors such as ageing of the population and an increased number of people with overweight ${ }^{[1]}$. Type 2 diabetes mellitus has been associated with an increased risk of several types of cancer ${ }^{[2,3]}$. One of the most important risk factors for both diseases is an increased body mass index (BMI). It is generally known that overweight is associated with an increased risk of type 2 diabetes mellitus mellitus ${ }^{[3]}$. Moreover, overweight has been associated with an increased risk of many cancer types ${ }^{[4]}$. Part of this effect could be explained by an increased cancer risk in patients with diabetes through insulin resistance because high insulin levels may have a growth promoting activity ${ }^{[5]}$. However, there is evidence that overweight is also associated with cancer through underlying mechanisms that are independent of insulin resistance ${ }^{[4]}$.

Drugs used for the treatment of type 2 diabetes mellitus might also be involved in the risk of developing cancer. In recent years, many studies have been focusing on the association between commonly prescribed drugs in type 2 diabetes mellitus and the risk of cancer ${ }^{[6]}$. Previous studies suggested a protective effect of metformin on cancer risk, while sulfonylurea derivatives and exogenous insulin were associated with a potentially increased cancer risk $^{[3,6,7]}$. However, the association between these drugs and the risk of cancer is complex since many factors are involved, and these factors might also interfere with each other. For example, sulfonylurea derivatives can lead to weight gain, overweight is associated with insulin resistance and type 2 diabetes mellitus, and overweight as well as type 2 diabetes mellitus as well as drugs used for type 2 diabetes mellitus are associated with cancer risk ${ }^{[2,8]}$.

The most frequently prescribed oral glucose lowering drugs are metformin and sulfonylurea derivatives. Large studies published in the late nineties indicated metformin as first choice drug for type 2 diabetes mellitus ${ }^{[9-11]}$. In these studies, the efficacy of metformin and sulfonylurea derivatives in lowering glycosylated haemoglobin (HbAlc) was equal. However, metformin therapy decreased the risk of any diabetes-related clinical endpoint, and was associated with less weight gain and less hypoglycaemic events in comparison to sulfonylurea derivatives. Since then, metformin is favoured over sulfonylurea derivatives, especially in obese patients. However, metformin is contra-indicated in those with loss of renal function with a creatinine clearance of less than $30 \mathrm{ml} / \mathrm{min}$. Therefore, physicians prescribe metformin and sulfonylurea derivatives to different patients.

In studies on the association between the use of metformin and cancer risk in comparison to the use of sulfonylurea derivatives, it is important to adjust for a potential difference in underlying factors that determine the choice of therapy (e.g. metformin or sulfonylurea derivative). In the current study, our objective was to investigate whether the determinants age, BMI, alcohol consumption and renal function were associated with the probability of being dispensed metformin or sulfonylurea derivatives as first drug therapy for type 2 diabetes mellitus.

\section{Methods}

\section{Setting}

This study was embedded in the Rotterdam Study, a prospective population-based cohort study of chronic diseases in the elderly population. All persons aged 55 years and over in the Ommoord district of Rotterdam, the Netherlands, were invited to participate $(\mathrm{n}=10,275)$. Of them, $7,983(78 \%)$ were enrolled between 1990 and 1993 to form the initial cohort (RS-1). The Medical Ethical Committee of the Erasmus Medical Center, Rotterdam, approved the study, and all participants gave informed consent. Participants have been continuously followed since then during follow-up rounds (1993-1995, 1997-1999, 20022004, 2009-2012). Furthermore, in 2000, an extended cohort was enrolled, the Rotterdam Study II (RS-II). 3,011 inhabitants entered the study and have been continuously followed since then. Detailed information on design, objectives and methods of this study have been described before ${ }^{[12,13]}$. For both cohorts, medication dispensing data were obtained from all seven fully computerized pharmacies in the Ommoord suburb. Information on all filled prescriptions from January $1^{\text {st }} 1991$ until February $1^{\text {st }} 2012$ were available and included information on the product name of the drug, the WHO's Anatomical Therapeutical Chemical code (ATC-code), the amount dispensed, the prescribed dosage regimen and the date of dispensing.

\section{Study population}

The study population consisted of all participants in the first and second cohort of the Rotterdam study (RS-I and RS-II) with a prescription for metformin or a sulfonylurea derivative between January $1^{\text {st }} 1991$ and December $1^{\text {st }} 2011$. Use of sulfonylurea derivatives or metformin was defined as a prescription of a drug from ATC-code 'A10'[14]. Available glucose-lowering drugs in ATC-codes 'A10BA' and 'A10BB' in the Rotterdam Study were metformin (A10BA02), glibenclamide (A10BB01), tolbutamide (A10BB03), glipizide (A10BB07), gliclazide (A10BB09), and glimepiride (A10BB12). Patients with a prescription for other glucose-lowering medication prior to the start of metformin or sulfonylurea derivative therapy were excluded. To ensure that all participants were incident users, a prescription free period of 90 days was obligatory and all metformin and sulfonylurea derivatives users with a first prescription before April $1^{\text {st }} 1991$ were excluded.

\section{Outcome and covariables}

The outcome of interest was starting with metformin or with a sulfonylurea derivative as first choice treatment of type 2 diabetes mellitus.

The determinants BMI and renal function were considered as factors influencing the choice for metformin versus sulfonylurea derivatives. Metformin use is associated with less weight gain and therefore it is likely that overweight subjects preferably start with metformin. Renal dysfunction (creatinine clearance $<30 \mathrm{ml} / \mathrm{min}$ ) is a contraindication for metformin therapy, and therefore patients initiating metformin are expected to have a better renal function than patients initiating sulfonylurea derivative therapy. Furthermore, we considered age and alcohol consumption in the analysis. Since metformin is first choice therapy in type 2 diabetes mellitus, the large studies in the late nineties, patients initiating metformin are expected to be relatively younger than patients initiating sulfonylurea derivatives. Excessive alcohol consumption is a contraindication for the use of metformin. Since the guidelines for treatment of type 2 diabetes mellitus have changed over time, we adjusted the analysis for calendar time. 
In the Rotterdam Study, during the center visits anthropmetric measures were performed by well-trained nurses. BMI was calculated as weight $(\mathrm{kg})$ divided by length $\left(\mathrm{m}^{2}\right)$. Information on alcohol consumption was obtained by an interview at home, and was expressed in number of alcohol consumptions per day. Serum creatinine was assessed by a nonkinetic alkaline picrate (Jaffe) method ${ }^{[15]}$. Creatinine clearance, as measure of renal function, was computed with the Cockcroft- Gault equation $^{[16]}$ corrected with a factor 0.9 and standardized for $1.73 \mathrm{~m}^{3}$ body surface area using the Dubois formula ${ }^{[17]}$ : GFR $=(140-$ age[years]) (weight $[\mathrm{kg}] \times 1.23)(0.85$ if female) (serum creatinine $[\mu \mathrm{mol} / \mathrm{L}])^{-1}(0.9)(1.73)(\text { weight }[\mathrm{kg}])^{-0.425}$ (height $\left.[\mathrm{cm}]\right)^{-0.725}$ $(0.007184)^{-1}$. Creatinine clearance generally exceeds GFR by $10 \%$ to $15 \%$ because of additional urinary creatinine excretion attributable to tubular secretion ${ }^{[18]}$. The Cockcroft-Gault estimate of GFR was therefore additionally corrected with a factor of 0.9. When participants received a first dispensing of metformin or sulfonylurea derivative, the most recent values of the determinants were retrieved and considered in the analysis.

\section{Statistical analysis}

Differences in baseline characteristics between incident metformin users and incident sulfonylurea derivative users were tested for significance with a t-test for continuous variables and $\mathrm{X}^{2}$-test for binary variables.

We performed logistic regression analyses to investigate whether the determinants age, BMI, alcohol consumption, and renal function were associated with the probability of initiating therapy with either metformin versus sulfonylurea derivatives. All variables were included in the model as continuous variables.

Metformin was introduced as first choice therapy in patients with type 2 diabetes mellitus with normal renal function after the large scale metformin studies in 1998/1999. Since in response to these studies the national prescription guideline for general practitioners was changed, analyses were stratified for patients initiating therapy before July $1^{\text {st }} 2000$ and patients initiating therapy after July $1^{\text {st }} 2000$. All analyses were performed using SPSS software (SPSS Inc., version 20.0, Chicago, Illinois, USA).

\section{Results}

Baseline characteristics of the study population are shown in table 1. We identified 639 incident users of metformin as first drug therapy and 934 incident users of sulfonylurea derivative as first drug therapy for type 2 diabetes mellitus between April $1^{\text {st }} 1991$ and December $1^{\text {st }} 2011$. The median start- date for metformin therapy was approximately 9 years later (November 2006) compared to sulfonylurea derivatives (August 1997). Starters of metformin had a higher BMI $(\mathrm{P}<.0001)$, were younger $(\mathrm{P}<.0001)$, and consumed more units of alcohol per day $(\mathrm{P}=.005)$ compared to starters of sulfonylurea derivatives.

The results of the logistic regression analysis on the probability of patients with type 2 diabetes mellitus starting with metformin versus sulfonylurea derivatives are shown in table 2. Calendar time was associated with the probability of starting with metformin versus sulfonylurea derivatives ( $\mathrm{P}$ - value $<$ .0001). Results are therefore presented stratified for those starting before and after July 1 st 2000 . In univariate analyses, in patients that started drugs for type 2 diabetes mellitus before July $1^{\text {st }} 2000$, lower age (OR 0.95; 95\%CI .92 - .98) and higher BMI (OR 1.16; 95\% CI $1.03-1.29$ ) were significantly associated with being dispensed metformin. Also in patients who started drugs for type 2 diabetes mellitus after July $1^{\text {st }} 2000$, lower age (OR $0.97 ; 95 \%$ CI .95 - .99) and higher BMI (OR 1.20; 95\% CI 1.13 - 1.27) were significantly associated with being dispensed metformin. For time frames, renal function nor alcohol consumption were associated with dispensing of metformin.

After further adjustment for the other potential confounders, only BMI was statistically significantly associated with the probability of dispensing metformin versus sulfonylurea derivatives as first prescription independently of calendar time (table 2). Starters before July $1^{\text {st }} 2000$ showed an OR of $1.19(95 \%$ CI $1.04-1.37)$ and starters after July $1^{\text {st }} 2000$ showed an OR of 1.23 (95\% CI $1.04-1.45)$ for BMI. In contrast with the univariate analyses, in the multivariate model, age showed no statistically significant association with the probability of starting with metformin versus sulfonylurea derivatives. Similar to the univariate analyses, in the adjusted analyses, null results were found for alcohol consumption and renal function.

Table 1: Baseline characteristics of the study population of incident metformin and sulfonylurea derivative users.

\begin{tabular}{|c|c|c|c|}
\hline & $\begin{array}{c}\text { Starters with } \\
\text { Metformin }(N=639)\end{array}$ & $\begin{array}{c}\text { Starters with } \\
\text { Sulfonylurea derivatives }(\mathrm{N}=\mathbf{9 3 4})\end{array}$ & $\mathbf{P}$ \\
\hline Age, years, mean(SD) & $69.2(10.8)$ & $71.3(10.7)$ & $<.0001$ \\
\hline Men, $\mathbf{N}(\%)$ & $288(45.1 \%)$ & $420(45.0 \%)$ & .968 \\
\hline BMI, $\mathrm{kg} / \mathrm{m}^{2}$, mean(SD) & $30.7(5.3)$ & $28.2(4.0)$ & $<.0001$ \\
\hline Alcohol consumption, units/day, mean(SD) & $1.40(1.5)$ & $1.16(1.7)$ & .005 \\
\hline Renal function: GFR, ml/min, mean(SD) (min - max) & $\begin{array}{c}74.5(19.0) \\
(40.1-145.3)\end{array}$ & $\begin{array}{c}75.8(19.7) \\
(20.7-164.6)\end{array}$ & .732 \\
\hline \multicolumn{4}{|l|}{ Start date first prescription } \\
\hline - Median & 09 Nov 2006 & 21 Aug 1997 & \\
\hline - First start date in database & 23 Aug 1991 & 02 Apr 1991 & \\
\hline - Last start date in database & 23 Nov 2011 & 18 Nov 2011 & \\
\hline Starters < 1 July 2000 (N) & 38 & 678 & \\
\hline Starters $\geq 1$ July 2000 (N) & 601 & 256 & \\
\hline
\end{tabular}

SD: standard deviation; BMI: body mass index; GFR: glomerular filtration rate 
Table 2: Results of the logistic regression analysis on the association between determinants and the probability of starting with metformin versus sulfonylurea derivatives - stratified on start date $<1$ July 2000 and start date $\geq 1$ July 2000.

\begin{tabular}{|c|c|c|c|c|c|c|c|c|}
\hline & \multicolumn{4}{|c|}{ Start date $<1$ July 2000} & \multicolumn{4}{|c|}{ Start date $\geq 1$ July 2000} \\
\hline & OR & $\boldsymbol{\beta}$ & $95 \% \mathrm{CI}$ & $\mathbf{P}$ & OR & $\boldsymbol{\beta}$ & $95 \% \mathrm{CI}$ & $\mathbf{P}$ \\
\hline \multicolumn{9}{|l|}{ Analysis per covariate* } \\
\hline - Age & 0.95 & -.050 & $.92-.98$ & .001 & 0.97 & -.030 & $.95-.99$ & $<.0001$ \\
\hline - BMI & 1.16 & .144 & $1.03-1.29$ & .011 & 1.20 & .180 & $1.13-1.27$ & $<.0001$ \\
\hline - Alcohol consumption & 0.89 & -.112 & $.70-1.14$ & .373 & 1.04 & .040 & $.91-1.19$ & .556 \\
\hline - Renal function & 0.96 & -.005 & $.96-1.03$ & .755 & 0.97 & -.035 & $.93-1.01$ & .081 \\
\hline \multicolumn{9}{|l|}{ Full model $\uparrow$} \\
\hline Age & 0.92 & -.082 & $.83-1.02$ & .118 & 0.95 & -.051 & $.87-1.04$ & .266 \\
\hline BMI & 1.19 & .176 & $1.04-1.37$ & .011 & 1.23 & .206 & $1.04-1.45$ & .017 \\
\hline Alcohol consumption & 1.26 & .228 & $.86-1.78$ & .202 & 0.96 & -.038 & $.49-1.89$ & .913 \\
\hline Renal function & 0.98 & -.019 & $.94-1.02$ & .374 & 0.95 & -.048 & $.91-1.01$ & .066 \\
\hline
\end{tabular}

* Model with covariate separately in the model, adjusted for calendar time. $†$ Full model with all covariates including calendar time in the model. OR: odds ratio; $\beta$ : beta; $\mathrm{CI}$ : confidence interval; BMI: body mass index. Bold values indicate statistically significant associations.

\section{Discussion}

In this population-based study, BMI was statistically significantly associated with an increased probability of dispensing of metformin as first drug therapy for type 2 diabetes mellitus. Patients starting on metformin therapy have a significantly higher BMI than patients starting on sulfonylurea derivatives.

This study demonstrates the importance of BMI in the choice of first therapy for type 2 diabetes mellitus. With regard to calendar time, the association between BMI and the probability of getting metformin versus sulfonylurea derivatives as first prescription was somewhat stronger in patients initiating therapy after July $1^{\text {st }} 2000$ than in patients initiating therapy before July $1^{\text {st }} 2000$. This is expected, since large studies in the late nineties indicated metformin as first choice therapy in type 2 diabetes mellitus patients, amongst others because of less weight gain with the use of metformin ${ }^{[9-11]}$. Before these studies, sulfonylurea derivatives were more often prescribed. In continuation of these studies the prescription guidelines for physicians were adapted, leading to metformin as first line therapy for type 2 diabetes mellitus, especially in those with overweight. Based on the contra- indication, one would also expect renal function to be an important determinant in the choice of therapy. However, in this study we could not demonstrate this. The mean renal function in the Rotterdam Study was about $75 \mathrm{ml} / \mathrm{min}$, while according to the Dutch prescription guidelines metformin is contraindicated in patient with a GFR $<30 \mathrm{ml} / \mathrm{min}$. Therefore, the majority of the population would not have a contra-indication for metformin ( $\mathrm{N}=4$ participants with a GFR $<30 \mathrm{ml} / \mathrm{min}$ ). However, renal function is not measured at every Rotterdam Study visit, and especially in the metformin group data on renal function was missing. This might also explain why we did not find an association between renal function and the probability of dispensing of metformin as initial therapy. As expected, metformin users were relatively younger, but age itself was not a statistically significant determinant in the choice of therapy after adjustment for other determinants.

Increased BMI is associated with an increased risk of type 2 diabetes mellitus as well as an increased risk of cancer ${ }^{[3,4]}$. However, this study showed that BMI is also associated with the probability of being dispensed metformin versus sulfonylurea derivatives. Therefore, when analyses are performed assessing the association between the use of these drugs and the risk of cancer, BMI should be considered as a confounder. Previous studies investigating the association between drugs used in type 2 diabetes mellitus and cancer risk suggested a protective effect of metformin on cancer risk, while sulfonylurea derivatives and exogenous insulin were associated with a potentially increased cancer risk $^{[3,6,7]}$. However, possible biological mechanisms explaining these associations are largely speculative and more research is needed to elucidate the true magnitude and direction of these associations. For example, the decreased risk of cancer in those using metformin compared with those using sulfonylurea derivatives could also be explained as an increased risk of cancer in users of sulfonylurea derivatives compared with users of metformin ${ }^{[19]}$. Moreover, although several studies on this topic adjusted for BMI or match on BMI ${ }^{[20-28]}$, there are also several studies that do not consider BMI as confounder ${ }^{[19,29-32]}$. The question remains whether in these studies lacking BMI adjustment and demonstrating a protective effect of metformin on cancer risk $^{[19,29-32]}$, indeed the protective effect persists when these studies adjust for BMI. In our study, metformin users had a higher BMI than sulfonylurea derivatives users, which makes metformin users hypothetically potentially more at risk for cancer than sulfonylurea derivative users with regard to BMI as risk factor. When studies demonstrated a protective effect on cancer risk for metformin users compared to sulfonylurea derivatives users without BMI adjustment, this protective effect might therefore change when BMI is considered as a confounder.

As discussed, the association between drugs used in type 2 diabetes mellitus, and the risk of cancer is complex and investigating this association requires accurate analysis methods to prevent biased results. Underlying mutual risk factors are involved and considering these factors as potential confounders or effect modifiers is obligatory. Furthermore, studies performing these kind of analyses are susceptible for 'reverse causation' where an undetected cancer causes changes in glucose and insulin metabolism leading to the onset of diabetes, and for 'detection bias' where cancer is more likely to be diagnosed in newly diagnosed patients with diabetes since they are more frequently 
contacting a physician. Last, 'depletion of susceptibles' should be considered where patients with diabetes already died of other causes and are less susceptible of being diagnosed with can$\operatorname{cer}^{[7,33]}$. Taking into account all these factors is of major importance and interest in future studies investigating the association between drugs used in type 2 diabetes mellitus and cancer risk, and will hopefully elucidate more insights in the true association. Given the results of the current study, we can add that these future studies should be adjusted for BMI.

However, limitations of the current study should be considered. Cohort studies are prone to selection bias, information bias and confounding. The risk of selection or information bias is unlikely since the Rotterdam Study is a population-based cohort study, in which data is collected prospectively without prior knowledge of the aim of this study. In the analysis, we adjusted for calendar time. In the Rotterdam Study, participants are visiting the center at every $4-5$ years of follow-up period and new data are collected. We considered the measurements of for example BMI and alcohol consumption which were the closest to the initial dispensing date. Unfortunately, we had missing data on renal function. Another limitation is that we were not able to filter out those who used metformin for other indications (e.g. polycystic ovarian disease). However, such diseases occur at a low frequency, and these indications are not registered in the Netherlands. Consequently, the number of those using metformin for indications other than diabetes most likely was too low to bias the risk estimates in our study.

\section{Conclusion}

In conclusion, BMI is associated with a higher probability of dispensing of metformin as first drug therapy for type 2 diabetes mellitus in comparison to sulfonylurea derivatives. Since BMI is associated with the risk of cancer as well as the risk of type 2 diabetes mellitus, studies analyzing the association between the use of metformin or sulfonylurea derivatives and the risk of cancer should also consider BMI as determinant in the analyses.

\section{Implications}

With rising prevalence of type 2 diabetes mellitus, health care educators/providers should also be aware of the long term complications of diabetes mellitus such as an increased risk of several types of cancer. BMI has been associated with an increased risk of type 2 diabetes mellitus as well as an increased cancer risk. Furthermore, studies have suggested a protective effect of metformin on cancer risk, while sulfonylurea derivatives and exogenous insulin were associated with a potentially increased cancer risk. Health care educators/providers should be aware of the complexity of these associations since all these factors may contribute differently to the risk of development of, for example, the diagnosis of cancer.

Data from this study demonstrate that BMI is an essential covariable in studies analyzing the association between the use of metformin or sulfonylurea derivatives and the risk of cancer. Knowledge about the correct confounders is essential to elucidate the direction of the effect of the oral glucose lowering drugs on cancer risk. Although several studies on this topic adjusted for BMI or match on BMI, there are also several studies that do not consider BMI as confounder. The question remains whether, in these studies lacking BMI adjustment, the effect might change when BMI is considered as a confounder. Due to the complex association between BMI, type 2 diabetes mellitus, drugs used for type 2 diabetes mellitus, and cancer risk, one should always consider BMI as a co-variable.

\section{Acknowledgements and Funding}

The contribution of inhabitants, general practitioners, and pharmacists of the Ommoord district to the Rotterdam Study is gratefully acknowledged.

The Rotterdam Study is supported by the Erasmus Medical Center and Erasmus University Rotterdam; the Netherlands Organization for Health Research and Development (ZonMW); the Research Institute for Diseases in the Elderly; the Ministry of Education, Culture and Sciences; the Ministry of Health Welfare and Sports; the European Commission and the Municipality of Rotterdam.

Conflict of Interest: The authors declare that they have no conflict of interest.

\section{References}

1. Wild, S., Roglic, G., Green, A., et. Global prevalence of diabetes: estimates for the year 2000 and projections for 2030. (2004) Diabetes Care 27(5): 1047- 1053.

2. Vigneri P, Frasca F, Sciacca L, Pandini G, Vigneri R. Diabetes and cancer. (2009) Endocr Relat Cancer 16(4): 1103-1123.

3. Giovannucci, E., Harlan, D.M., Archer, M.C., et al. Diabetes and cancer: a consensus report. (2010) Diabetes Care 33(7): 1674-1685.

4. Renehan, A.G., Tyson, M., Egger, M., et al. Body-mass index and incidence of cancer: a systematic review and meta-analysis of prospective observational studies. (2008) Lancet 371(9612): 569-578.

5. Foulstone, E., Prince, S., Zaccheo, O., et al. Insulin-like growth factor ligands, receptors, and binding proteins in cancer. (2005) J Pathol 205(2):145-153.

6. Smith, U., Gale, E.A. Does diabetes therapy influence the risk of cancer? (2009) Diabetologia S52(9): 1699-1708.

7. Johnson, J.A., Carstensen, B., Witte, D., et al. Diabetes and cancer (1): evaluating the temporal relationship between type 2 diabetes and cancer incidence. (2012) Diabetologia 55(6): 1607-1618.

8. Vigneri, R. Diabetes: diabetes therapy and cancer risk. (2009) Nat Rev Endocrinol 5(12): 651-652.

9. UK Prospective Diabetes Study (UKPDS) Group. Effect of intensive blood-glucose control with metformin on complications in overweight patients with type 2 diabetes (UKPDS 34). (1998) Lancet 352(9131): 854-865.

10. UK Prospective Diabetes Study (UKPDS) Group. Intensive blood-glucose control with sulphonylureas or insulin compared with conventional treatment and risk of complications in patients with type 2 diabetes (UKPDS 33). (1998) Lancet 352(9131): 837- 853.

11. Johansen, K. Efficacy of metformin in the treatment of NIDDM. Meta-analysis. (1999) Diabetes Care 22(1): 33-37.

12. Hofman, A., Grobbee, D.E., de Jong, P.T., et al. Determinants of disease and disability in the elderly: the Rotterdam Elderly Study. (1991) Eur J Epidemiol 7(4): 403-422.

13. Hofman, A., Darwish Murad, S., van Duijn, C.M., et al. The Rotterdam Study: 2014 objectives and design update. (2013) Eur J Epidemiol 28(11): 889-926.

14. Complete ATC index 2010. Collaborating Centre for Drugs Statistics Methodology Web site. (2010) WHO.

15. Perrone, R.D., Madias, N.E., Levey, A.S. Serum creatinine as an index of renal function: new insights into old concepts. (1992) Clin Chem 38(10): 1933-1953. 
16. Cockcroft, D.W., Gault, M.H. Prediction of creatinine clearance from serum creatinine. (1976) Nephron 16(1): 31-41.

17. Wang, Y., Moss, J., Thisted, R. Predictors of body surface area. (1992) J Clin Anesth 4(1): 4-10.

18. Vervoort, G., Willems, H.L., Wetzels, J.F. Assessment of glomerular filtration rate in healthy subjects and normoalbuminuric diabetic patients: validity of a new (MDRD) prediction equation. (2002) Nephrol Dial Transplant 17(11): 1909-1913.

19. Ruiter, R., Visser, L.E., van Herk-Sukel, M.P., et al. Lower risk of cancer in patients on metformin in comparison with those on sulfonylurea derivatives: results from a large population-based follow-up study. (2012) Diabetes Care 35(1): 119-124.

20. Evans, J.M., Donnelly, L.A., Emslie-Smith, A.M., et al. Metformin and reduced risk of cancer in diabetic patients. (2005) BMJ 330(7503): 1304-1305.

21. Libby, G., Donnelly, L.A., Donnan, P.T., et al. New users of metformin are at low risk of incident cancer: a cohort study among people with type 2 diabetes. (2009) Diabetes Care 32(9): 1620-1625.

22. Monami, M., Colombi, C., Balzi, D., et al. Metformin and cancer occurrence in insulin-treated type 2 diabetic patients. (2011) Diabetes Care 34(1): 129-131.

23. Monami, M., Lamanna, C., Balzi, D., et al.. Sulphonylureas and cancer: a case-control study. (2009) Acta Diabetol 46(4): 279-284.

24. Bodmer, M., Meier, C., Krahenbuhl, S., et al. Long-term metformin use is associated with decreased risk of breast cancer. (2010) Diabetes Care 33(6): 1304-1308.
25. Donadon, V., Balbi, M., Mas, M.D., et al. Metformin and reduced risk of hepatocellular carcinoma in diabetic patients with chronic liver disease. (2010) Liver Int 30(5): 750-758.

26. Li, D., Yeung, S.C., Hassan, M.M., et al. Antidiabetic therapies affect risk of pancreatic cancer. (2009) Gastroenterology 137(2): 482-488. 27. Wright, J.L., Stanford, J.L. Metformin use and prostate cancer in Caucasian men: results from a population-based case-control study. (2009) Cancer Causes Control 20(9): 1617- 1622.

28. Chlebowski, R.T., McTiernan, A., Wactawski-Wende, J., et al. Diabetes, metformin, and breast cancer in postmenopausal women. (2012) J Clin Oncol 30(23): 2844-2852.

29. Currie, C.J., Poole, C.D., Gale, E.A. The influence of glucose-lowering therapies on cancer risk in type 2 diabetes. (2009) Diabetologia 52(9): 1766-1777.

30. Hassan, M.M., Curley, S.A., Li, D., et al. Association of diabetes duration and diabetes treatment with the risk of hepatocellular carcinoma. (2010) Cancer 116(8): 1938-1946.

31. Lee, M.S., Hsu, C.C., Wahlqvist, M.L., et al. Type 2 diabetes increases and metformin reduces total, colorectal, liver and pancreatic cancer incidences in Taiwanese: a representative population prospective cohort study of 800,000 individuals. (2011) BMC Cancer 11:20.

32. Hsieh, M.C., Lee, T.C., Cheng, S.M., et al. The influence of type 2 diabetes and glucose-lowering therapies on cancer risk in the Taiwanese. (2012) Exp Diabetes Res 2012: 413782.

33. Renehan, A.G., Yeh, H.C., Johnson, J.A., et al. Diabetes and cancer (2): evaluating the impact of diabetes on mortality in patients with cancer. (2012) Diabetologia 55(6): 1619-1632.
Ommega Online Publishers

Journal Title: Journal of Diabetes and Obesity (JDO)

Journal Short Name: J diabetes Obes
Journal ISSN: 2356-0494

E-mail: diabetes@ommegaonline.com

Website: www.ommegaonline.org 